Physics

Physics Research Publications

\title{
Experimental constraints on strangelets and other exotic nuclear matter
}

D. Javorsek E. Fischbach

D. Elmore 


\title{
Experimental constraints on strangelets and other exotic nuclear matter
}

\author{
D. Javorsek II* \\ Department of Physics, Purdue University, West Lafayette, Indiana 47907 \\ and 80th Flying Training Wing, United States Air Force, Sheppard Air Force Base, Texas 76311 \\ E. Fischbach ${ }^{\dagger}$ and D. Elmore* \\ Department of Physics, Purdue University, West Lafayette, Indiana 47907 \\ (Received 19 October 2002; published 25 February 2003)
}

\begin{abstract}
A reanalysis of data from a recent search for ultraheavy isotopes of gold and iron leads to new constraints on several classes of exotic objects. These include strangelets, MEMOs (metastable exotic multihypernuclear objects), and CHAMPs (charged massive particles) which may have been present in the data, but which could have nonetheless gone undetected due to the design of the original experiment. As a result of the new analysis we are able to greatly enlarge the exclusion regions for exotic particles of mass $M$ and charge $Z$, and provide limits as low as $10^{-11}$ for small $M / Z$, and $10^{-7}$ for $M / Z$ up to 120 .
\end{abstract}

DOI: 10.1103/PhysRevD.67.034015

PACS number(s): 12.38.Mh, 25.75.Gz

\section{INTRODUCTION}

In a recent series of papers, results were reported from a search for superheavy isotopes of gold and iron with masses up to $1.7 \mathrm{TeV} / c^{2}$ and $0.65 \mathrm{TeV} / c^{2}$, respectively [1-4]. These results were then used to set limits on the abundance of strongly interacting neutral massive particles (SIMPs) bound to gold or iron nuclei, noting that such SIMP-nucleus bound states would appear in this experiment as anomalously heavy isotopes of $\mathrm{Au}$ or Fe. The object of the present paper is to generalize the analysis in Refs. [1-4] so as to allow bounds to be set on other forms of exotic matter. Specifically, we focus on three classes of exotic objects which we describe below: strangelets, metastable exotic multihypernuclear objects (MEMOs), and charged massive particles (CHAMPs). As we will show in the ensuing discussion, the hypothesized properties of strangelets, MEMOs, and CHAMPs necessitate reanalyzing the raw data from the experiment described in Refs. [1-4], which only searched for neutral SIMPs.

The limits quoted in Refs. [1-4] were derived assuming that the massive particle had an overall nuclear charge of $Z$ $=79$. However, the exotic objects to be described below, strangelets, MEMOs, and CHAMPs, require a complete reanalysis of the raw data in Refs. [1-4], since these objects need not have $Z=79$. The restriction to $Z=79$ is significant, since the results obtained during the experiment and the subsequent analysis depended on the stripping efficiency $\eta$ and the detector calibrations of the Purdue accelerator mass spectrometer (AMS), which in turn are a function of $Z$.

Consider strangelets, for example, whose properties we discuss in detail in Sec. II A. For present purposes strangelets would appear in our experiment as objects with anomalously large baryon number-to-charge $(A / Z)$ or mass-to-

\footnotetext{
*Email address: javorsek@hotmail.com

${ }^{\dagger}$ Corresponding author. Email address:

ephraim@physics.purdue.edu

${ }^{\ddagger}$ Email address: elmore@purdue.edu
}

charge $(M / Z)$ ratios. Since the stripping efficiency $\eta$ depends on $M$ as well as on $Z$, it follows that strangelets will occupy a region in parameter space $\eta=\eta(M, Z)$ which is not accessed by ordinary matter. For this reason conventional software used to calculate $\eta$ must be adapted to apply to strangelets. Similar considerations apply to MEMOs which would also appear in the experiment of Refs. [1-4] with values of $M$ and $Z$ not covered by our previous analysis.

\section{MOTIVATION}

\section{A. Strangelets}

Various theoretical considerations suggest that strange quark matter (SQM) may exist, which would consist of objects containing roughly equal numbers of $u, d$, and $s$ quarks. (See Refs. [5,6] for recent reviews.) Objects composed of SQM could range from small (strangelets) to large (strange stars), and might also constitute a component of cosmic radiation in the form of quark nuggets [5-7]. The possibility that strange stars may in fact exist received a boost recently based on data obtained from the Chandra X-Ray Observatory $[8,9]$. Although not conclusive, two separate analyses of the Chandra data suggested the presence of collapsed objects smaller than neutron stars, which is compatible with theoretical expectations for strange stars.

From the point of view of the present analysis, strangelets would appear as nuclei which are neutral, or close to neutral, despite having a large baryon number. This follows by noting that the sum of the charges on a $u, d$, and $s$ quark is zero, and hence any object containing roughly equal numbers of $u, d$, and $s$ quarks would be approximately neutral. This observation, along with various scenarios for strangelet production, form the basis for the present analysis.

In the first scenario, we assume that strangelets are in fact the true ground state of QCD, as postulated by Witten [7], and hence fragments of SQM created in the big bang could remain in trace quantities as part of our natural environment. As a result, back scattering $[10,11]$ and mass spectrometry experiments $[1,2,12,13]$ may be sensitive to such strangelets. Similarly, cosmic ray data have also been analyzed for the 
presence of strangelets [14-16], and possible signals for SQM nuggets have been proposed from seismic events [17].

A second scenario for strangelet production is associated with the intense energy created in heavy-ion collisions in which an intermediate quark-gluon plasma (QGP) is formed. The QGP is hypothesized to be rich in $u$ and $d$ quarks and to have initial strangeness of zero. However, as the plasma expands and cools it emits not only hadronized pions, but also hadronized kaons $\left(\mathrm{K}^{+}, \mathrm{K}^{0}\right)$ which are made of $\bar{s}$ quarks, thus leaving the QGP with a net strangeness. Since $\mathrm{K}^{+}$and $\mathrm{K}^{0}$ form more readily than their respective antiparticles $\mathrm{K}^{-}$ and $\overline{\mathrm{K}}^{0}$ in the presence of a plasma rich in $u$ and $d$ quarks, it follows that $\bar{s}$ quarks are depleted preferentially from the QGP compared to $s$ quarks, which is why the QGP may end up with a nonzero strangeness. In this scenario strangelets are essentially the cooled remnants of the QGP [6]. The third scenario for strangelet production is known as the coalescence model in which the products of the nucleus-nucleus collision form a composite state which fuses to form a strangelet [18]. Several experiments have been performed at heavy-ion colliders at the BNL Alternating Gradient Synchrotron (AGS) [19-24] and CERN Super Proton Synchrotron (SPS) [25] searching for strangelets by their large mass to charge ratio $(A / Z)$.

\section{B. MEMOs}

Relativistic heavy-ion collisions like those produced at the BNL AGS are also expected to yield tens of hyperons per collision [26]. This in turn creates an environment which is favorable to the formation of metastable exotic multihypernuclear objects (MEMOs). Schaffner, Greiner, and Stöcker [26] show that multi- $\Lambda$ hypernuclei are more strongly bound than normal nuclei using the relativistic mean-field model (a model which has been proven to give good descriptions of normal nuclei as well as $\Lambda$ hypernuclei). As a result, certain MEMOs may produce signals which would be similar to the strangelets discussed above $[27,28]$. Several MEMOs along with their properties are tabulated in Ref. [26].

\section{CHAMPs}

We consider first the case of a charged SIMP $\mathrm{X}^{ \pm Q}$ (with charge $\pm Q$ ) bound to a nucleus having a charge $Z$. (When $Q= \pm 1$ these particles are called charged massive particles, CHAMPs $[29,30]$, whose constraints may be found in Ref. [31].) In the experiments of Refs. [1-4], if $Z \pm Q=79$ then the resulting nucleus would be indistinguishable from a nucleus ${ }_{79} \mathrm{X}$ with mass $M_{X}$ formed from a neutral SIMP bound to ${ }_{79} \mathrm{Au}$. Since this was the case analyzed in Refs. [1-4], it follows that the limits obtained there apply immediately to charged SIMPs with arbitrary $Q$ attached to appropriate nuclei.

\section{Other exotic particles}

Finally we note that other types of exotic nuclear matter could have been present in the samples analyzed in the experiment of Refs. [1-4]. This experiment would have de- tected any nucleus having an anomalously large mass, or a mass that cannot be identified with a known isotope. This includes nuclei formed by the binding of exotic particles to a conventional nucleus, as well as a nucleus containing only protons and neutrons but having an unexpectedly large mass. In particular, our experiment constrains the hypothetical doubly magic superheavy nucleus ${ }^{310} \mathrm{G}(Z=126, N=184)$ [27].

\section{SAMPLES}

In this section we discuss the implications of our reanalysis of the data from a recent experiment by Javorsek et al. [1] carried out at the Purdue Rare Isotope Measurement Laboratory (PRIME Lab). In the PRIME Lab experiment, much effort was devoted to obtaining unique samples which would enhance the possibility of finding exotic nuclei. While all samples were sensitive to primordial exotic nuclear objects, two samples are particularly relevant for the discussion at hand: the first is a sample flown on board the Long Duration Exposure Facility (LDEF) satellite, and the second was obtained from the beam dump of experiment E878 run at the Brookhaven AGS. A discussion of the implications of the LDEF sample for dark matter may be found in Refs. [1-4]. Of more immediate interest to the present paper are the results from the AGS sample since they effectively combine two major methods of searching for terrestrial strangelets.

In E878 an Au beam was incident on an Au target resulting in more than $2 \times 10^{12} \mathrm{Au}+\mathrm{Au}$ collisions at a beam energy of $10.8 A \mathrm{GeV}$ [19]. E878 searched for strangelets with a spectrometer designed to detect the ejected products of the $\mathrm{Au}+\mathrm{Au}$ collision, and was sensitive to strangelets of charges $Z=-3,-2,-1,+1,+2,+3$ up to a mass of $A=30$. Due to the design of the experiment, it was unable to detect either neutral strangelets or any strangelets which would have remained trapped in the target. The data reported in Refs. [1-4] analyzed the target material from E878 for anomalous nuclei.

If the conjecture that $\mathrm{SQM}$ is the true ground state of QCD [7] is correct then a strangelet would be an eigenstate of SU(3) and would be stable. This stability arises from the introduction of the third flavor ( $s$ quark) which in turn reduces the energy of SQM relative to the usual two flavor system composed of $u$ and $d$ quarks. This extra flavor provides an added Fermi potential well and makes it possible to increase the spatial concentration of quarks, which reduces the total SQM energy [5].

Since the strangelet is an eigenstate of $\mathrm{SU}(3)$ it is either an isoscalar, an isospinor, or an isovector. If the strangelet is an isoscalar then the strangelet-nucleus force would be attractive, and it would bind to the nuclei of ordinary elements. This binding will depend on the strangelet-nuclear potential and could preferentially bind to high- $Z$ nuclei [1]. Since strangelet remnants of the big bang would exist in only trace concentrations, the experiment of Hemmick et al. [12] may not have been sensitive enough to find primordial strangelets, especially if strangelets bind preferentially to heavy nuclei. In the PRIME Lab experiment [1-4] we circumvent these two problems by searching for strangelets bound to a high- $Z$ nucleus, $\mathrm{Au}$, and by using the target $\mathrm{Au}$ from E878 at the 


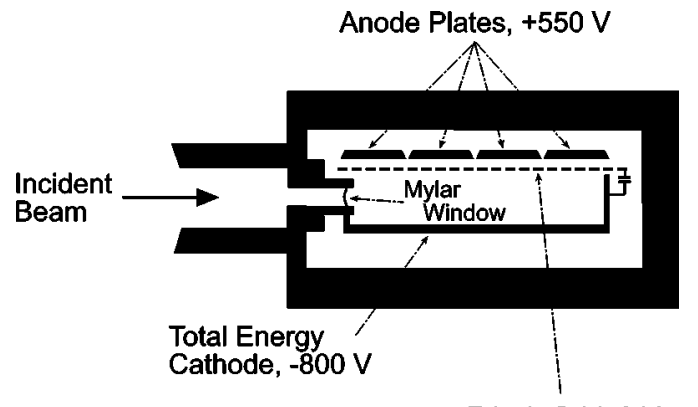

Frisch Grid, 0 V

FIG. 1. Schematic cross section of the PRIME Lab gas ionization detector. See the text for further details.

AGS which came from an environment favorable for strangelet production.

If neutral strangelets or those with $Z>3$ had been produced in the E878 experiment, they would have gone undetected. It is possible that some would have remained trapped in the target material and would then be visible in the PRIME Lab experiment. However, the data reported by Javorsek et al. [1] provides constraints on neutral strangelets only. Following a reanalysis of the PRIME Lab data, we found that changing the acceptable range of energy deposited in various regions of the gas ionization detector allows sensitivities to strangelets of $Z \geqslant 14$. This limit arises from the high charge states run in the experiment.

\section{REANALYSIS OF THE DATA}

In the experiment of Javorsek et al. [1-4], the samples were analyzed for a range of different terminal voltage settings $V_{T}$ at the PRIME Lab accelerator mass spectrometer (AMS). (For a description of the PRIME Lab AMS see Ref. [2].) This range of $V_{T}$ settings allowed all masses to be accessed in a scan from $2.7 \mathrm{GeV} / c^{2}$ to $1.67 \mathrm{TeV} / c^{2}$. Following the electrostatic and magnetostatic selection for the ion of interest at a given mass step, the particle passed from the beam pipe through a thin Mylar film into a gas ionization detector, which was filled with propane gas. The ions produce electron-ion pairs when they interact with the gas, and these pairs separate in the presence of a low transverse electric field, thus inducing voltage signals on the cathode and anode (see Fig. 1). For each $V_{T}$ step, a histogram of the number of events versus collected charge was created for each of the anode plates and cathode chamber. This allowed a determination of the energy deposited along the ion path (from the anode), as well as the total energy (from the cathode). Once the energy spectra were recorded in the detector, the AMS was reconfigured for the next value of $V_{T}$ and hence for the next mass step.

Energetic ions in the detector lose energy through interactions with the propane gas, eventually resulting in an ion with an equilibrium number of electrons bound to a nucleus of charge $Z$. This equilibrium state depends directly on both $Z$ and the incident energy. As a result, ions with the same energy but different $Z$ lose energy at different rates, and deposit different fractions of their total energy along corresponding segments of the ion path. Segmenting the anode
TABLE I. Peak channels for three representative Au beam energies, $\mathrm{E}_{b} . \Delta \mathrm{E}_{1}, \Delta \mathrm{E}_{2}$, and $\Delta \mathrm{E}_{3}$ denote the segmented anode plates on which the particles were detected, with $\Delta \mathrm{E}_{1}$ being closest to the Mylar window and $\Delta \mathrm{E}_{3}$ farthest away. Each plate has 1024 channels, and the channel number increases linearly with energy. For the cathode the single plate measures the total energy $E$.

\begin{tabular}{ccccc}
\hline \hline \multirow{2}{*}{ Beam energy } & \multicolumn{3}{c}{ Anode plate channel } & Cathode channel \\
$\mathrm{E}_{b}(\mathrm{MeV})$ & $\Delta \mathrm{E}_{1}$ & $\Delta \mathrm{E}_{2}$ & $\Delta \mathrm{E}_{3}$ & Total $E$ \\
\hline 22.9 & 459 & 375 & 387 & 852 \\
20.0 & 408 & 345 & 334 & 714 \\
16.8 & 336 & 303 & 83 & 522 \\
\hline \hline
\end{tabular}

thus provides us with knowledge of how each particle deposited its energy along its path and, if calibrated properly, this leads to a determination of $Z$. In the experiment of Refs. [1-4] the detector was calibrated for $Z=79$ by running $\mathrm{Au}$ at different energies, and then recording the channels where the peaks were observed for each plate. Table I gives the total beam energies as well as the peak Au channels on each plate. (Here the plate denoted by $\Delta \mathrm{E}_{1}$ is that closest to the Mylar window and $\Delta \mathrm{E}_{3}$ is the plate farthest away.) The pressure of the propane gas was chosen such that the highest energy beam would stop in the $\Delta \mathrm{E}_{3}$ plate. As the beam energy decreases, the particles do not penetrate as deeply into the detector as is evidenced by the sharper decline in the peak channel for the $\Delta \mathrm{E}_{3}$ plate.

Following this channel-to-energy calibration two different procedures were employed to analyze the results: First a histogram was produced that included the energies of all particles which entered the detector at a given $V_{T}$ step. Since the electromagnetic elements in the AMS beam line separated particles based on their mass-to-charge ratio $M / q$, and not exclusively on their mass (see Ref. [2] for further discussion), it follows that different particles with the same $M / q$ will pass through the electromagnetic elements and reach the detector. Thus the detector calibration discriminates among particles with the same $M / q$ based on their different energy spectra as reflected by the signals in the anode and cathode channels. This allowed us to determine which peak in the detector was the particle of interest, and to differentiate it from a normal atom in a different charge state masquerading as an exotic particle. For different $V_{T}$ steps we then anticipated the peak channel (i.e. energy) where the exotic particle (with a presumed mass $M$ ) would appear, and in turn ignored all peaks in different channels (i.e. at different energies and different $Z$ 's). For additional details on the experimental procedures see Refs. [1-4].

Unfortunately, there were several cases of strong contamination from peaks with energies close to the predicted energy of the exotic particle, and for these a second procedure was employed. Since each peak has associated with it a characteristic width (which arises from small $V_{T}$ fluctuations as well as from window impurities, energy straggling, etc.) it is possible for the tail of a nearby contaminating peak to swamp a small peak from an exotic particle, which we expect to be present in trace amounts. In order to eliminate this effect we introduced a process of "gating," which con- 
strained the energy spectrum of each plate. To accomplish this we created a program which only recorded counts with the proper fraction of the ion's total energy deposited on each plate. This program created a "window" or "gate" that was centered on the expected peak channel with a width equal to the full width at half maximum of the Au peak used in the calibration process. Only particles with energies that fell within the gates of all three plates were counted in the final result, which selected only those counts with the predetermined value of $Z$.

In the previous experimental results [1-4], the gating procedure described above was performed for $Z=79$ only. In the present reanalysis of the data, we modified the program to search for potential exotic particles with different values of $Z$ in order to provide constraints on exotic particles which were not neutral (as assumed in Ref. [2]).

The observation that atoms with different $Z$ arrive at different equilibrium charge states when introduced to a medium also affects the experimental results by changing the accelerator transmission efficiencies. In the AMS a negatively charged ion is created and accelerated by a large potential difference (the terminal voltage, $V_{T}$ ). At the terminal of the accelerator the ions are introduced to an Ar gas "stripper." This removes electrons and creates a positively charged ion which is then accelerated away from the terminal. This "tandem" acceleration produces the energies needed to separate the different ions based on their mass, charge, and energies, and also removes potential molecular contaminants.

As a result of the dynamics of the charge changing process that occurs in the stripper the negatively charged ions that enter the terminal exit with a distribution of different charge states. The number of ions which end up in the desired charge state is characterized by the stripper transmission efficiency $\eta$ for that charge state. Since the sensitivity of such AMS experiments requires an understanding of the dependence of $\eta$ on $V_{T}$, this question has been extensively studied in the literature [32]. It has been shown that $\eta\left(V_{T}\right)$ can be expressed phenomenologically in the form

$$
\eta=P_{1} \exp \left[\frac{P_{2}\left(V_{T}-P_{3}\right)^{2}}{1+P_{4}\left(V_{T}-P_{3}\right)}\right]
$$

where the constants $P_{1} \ldots P_{4}$ are determined empirically. In the present experiment we calibrated the stripper by running both $\mathrm{Au}$ and $\mathrm{Fe}$ at several terminal voltages, and then used these data to extract $P_{1} \ldots P_{4}$. Figure 2 displays the results for both $\mathrm{Au}$ and $\mathrm{Fe}$ with the values of the constants given in Table II. As a result this study provides us with the ability to interpolate $\eta$ for ions with different $\mathrm{Z}$.

From the table it appears that the most significant difference among nuclei with different $Z$ is the overall coefficient $P_{1}$ of the Gaussian, which can differ by an order of magnitude from one nucleus to another. However, there are other competing effects which determine the final sensitivity $\mathrm{X} / \mathrm{Au}$, such as the dependence of the stripping efficiency on the mass and velocity of the incident ions. (See Ref. [3] for further discussion.) From Ref. [2] the sensitivity $X / \mathrm{Au}$ is given by

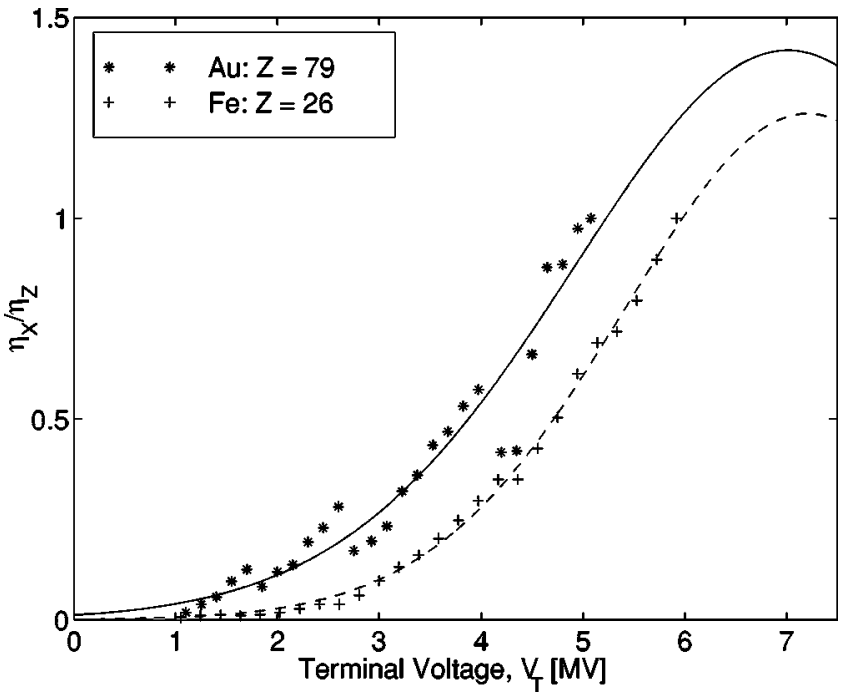

FIG. 2. Normalized transmission efficiency for both ${ }_{79} \mathrm{Au}$ and ${ }_{26} \mathrm{Fe}$ as a function of the terminal voltage $V_{T}$ in MV for charge state $q=+9$.

$$
\frac{X}{\mathrm{Au}}=\left(2.672 \times 10^{-12}\right) \frac{q R}{I_{\text {det }}}\left[\frac{\eta_{A u}}{\eta_{X}(M, Z)}\right] .
$$

Here $q$ is the charge state, $R$ is the observed count rate in counts per minute, and $I_{d e t}$ is the beam current measured at the detector Faraday cup. For a derivation of this equation see Ref. [2]. Our limits on exotic particles were obtained from Eq. (2) which is a modified version of the expression used to calculate the sensitivity in Refs. [1-4], coupled with the newly determined values of the stripper efficiencies for different $\mathrm{Z}$. We were able to return to the existing data and simply search the energy spectra for peaks which would have been rejected by the previous search, but which we would now identify as having the characteristics of a strangelet or other massive nuclear object. All peaks found via the regating procedure were easily explainable, and this allowed us to set several new constraints on the presence of strangelets in experiments like E878 at BNL. Our results are shown in Fig. 3 and are presented in a format similar to other strangelet searches where the sensitivity is plotted against the $M / Z$ ratio. Recall that the lower limit of $Z=14$ arises from the high charge states of up to 14 used in the original experiment.

\section{CONCLUSIONS}

We have shown that following a reanalysis of the raw data obtained originally by Javorsek et al. [1,2] we are able to

TABLE II. Coefficients for the stripper transmission efficiency curve fit at charge state $q=+9$ for both ${ }_{79} \mathrm{Au}$ and ${ }_{26} \mathrm{Fe}$. The definition of these parameters can be found in Eq. (1).

\begin{tabular}{ccccc}
\hline \hline Atom & $P_{1}$ & $P_{2}$ & $P_{3}[M V]$ & $P_{4}$ \\
\hline${ }_{79} \mathrm{Au}$ & 0.066 & -0.12 & 7.014 & -0.028 \\
${ }_{26} \mathrm{Fe}$ & 0.67 & -0.16 & 7.207 & -0.026 \\
\hline \hline
\end{tabular}




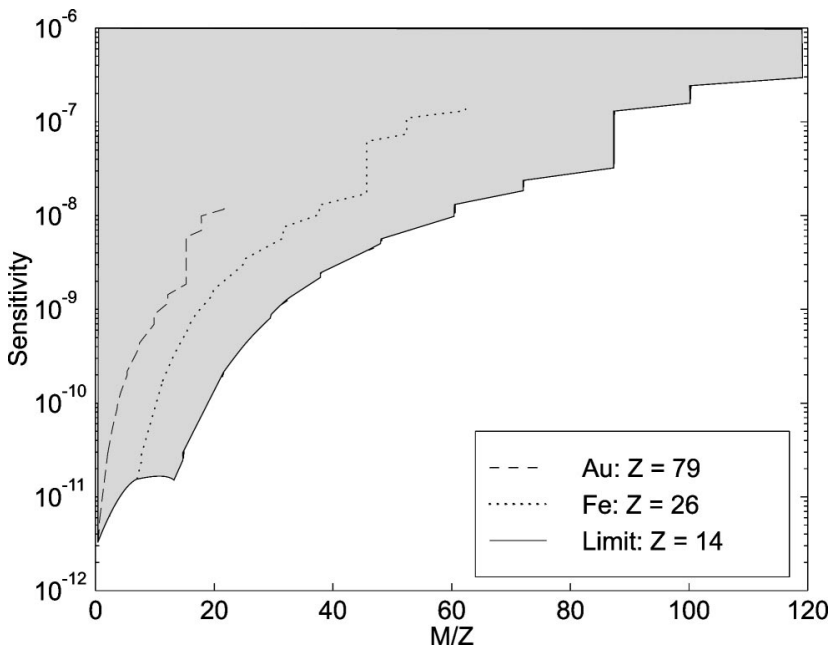

FIG. 3. Sensitivity, $X / Z$, of the AGS E878 sample as a function of $M / Z$ for three representative values of $Z$. The shaded region contained within the solid lines is excluded by the PRIME Lab results at the $95 \%$ confidence level. A similar exclusion region may be created for each of the other samples, although the AGS and LDEF samples come from environments that are most likely to contain exotic nuclear matter.

constrain the possible properties of strangelets, MEMOs, and CHAMPs. In fact, the samples examined (primarily from E878 at the AGS) should have enhanced the possibility of strangelet detection, since they came from an environment favorable to the formation of exotic objects. Since the limits provided in this paper are for a generic particle with mass $M$ and nuclear charge $Z$, one may interpret $M$ and $Z$ as either the properties of a free strangelet or as those of a bound system consisting of a strangelet with an ordinary nucleus (see Sec. II C).

This paper sets limits on the existence and properties of particles such as strangelets, MEMOs, and CHAMPs at sensitivities comparable to the previous AMS experiment [13].
TABLE III. Comparisons of the $M / Z$ and sensitivities for different experiments.

\begin{tabular}{lcc}
\hline \hline Experiment & $M / Z$ range & Reference \\
\hline E878 $(\mathrm{Au}+\mathrm{Au})$ & $-20 \leqslant M / Z \leqslant-2$ & {$[19]$} \\
& $2 \leqslant M / Z \leqslant 15$ & {$[19]$} \\
AMS $(\mathrm{He})$ & $21 \leqslant M / Z \leqslant 41$ & {$[13]$} \\
E886 $(\mathrm{Si}, \mathrm{Au}+\mathrm{Pt})$ & $-12 \leqslant M / Z \leqslant-1$ & {$[20]$} \\
E864 $(\mathrm{Au}+\mathrm{Pt})$ & $2 \leqslant M / Z \leqslant 12$ & {$[20]$} \\
NA52 $(\mathrm{Pb}+\mathrm{Pb})$ & $-100 \leqslant M / Z \leqslant-2$ & {$[21,23]$} \\
& $2 \leqslant M / Z \leqslant 100$ & {$[22,23]$} \\
PRIME Lab & $-100 \leqslant M / Z \leqslant-2$ & {$[25]$} \\
\hline \hline
\end{tabular}

(Since the AMS experiments are configured differently from the collision experiments [19-25], a direct comparison of the sensitivities of each type of experiment is not meaningful.) Table III presents a summary of the range of $M / Z$ values from different searches for strangelets. By extending the range of $M / Z$ up to nearly $\cong 120$, we have significantly enlarged the exclusion region in parameter space for strangelets.

\section{ACKNOWLEDGMENTS}

We are indebted to S. Aronson and M. Bennett for supplying us with the E878 samples, and to F. Hörz and NASA for the LDEF sample. This work was supported in part by the U.S. Department of Energy under Contract No. DE-AC0276ER01428, and PRIME Lab is supported by National Science Foundation Grant No. 9809983-EAR. The views expressed in this paper are those of the authors and do not reflect the official policy or position of the U.S. Air Force, U.S. Department of Defense, or the U.S. Government.
[1] D. Javorsek II, D. Elmore, E. Fischbach, D. Granger, T. Miller, D. Oliver, and V. Teplitz, Phys. Rev. Lett. 87, 231804 (2001).

[2] D. Javorsek II, D. Elmore, E. Fischbach, D. Granger, T. Miller, D. Oliver, and V. Teplitz, Phys. Rev. D 65, 072003 (2002).

[3] D. Javorsek II, Ph.D. thesis, Purdue University, 2001.

[4] D. Javorsek II, D. Elmore, E. Fischbach, T. Miller, D. Oliver, and V. Teplitz, Phys. Rev. D 64, 012005 (2001).

[5] J. Madsen, astro-ph/9809032.

[6] R. Klingenberg, J. Phys. G 27, 475 (2001).

[7] E. Witten, Phys. Rev. D 30, 272 (1984).

[8] P. Slane, D.J. Helfand, and S.S. Murray, astro-ph/0204151.

[9] J.J. Drake et al., Astrophys. J. 572, 996 (2002).

[10] M. Brugger et al., Nature (London) 337, 434 (1989).

[11] M.C. Perillo Isaac et al., Phys. Rev. Lett. 81, 2416 (1998).

[12] T.K. Hemmick et al., Phys. Rev. D 41, 2074 (1990).

[13] J. Vandegriff et al., Phys. Lett. B 365, 418 (1996).
[14] S. Banerjee, S.K. Ghosh, S. Raha, and D. Syam, Phys. Rev. Lett. 85, 1384 (2000).

[15] M. Rybczynski, Z. Wlodarczyk, and G. Wilk, Acta Phys. Pol. B 33, 277 (2002).

[16] L. Masperi, astro-ph/0202096.

[17] D. Anderson, E. Herrin, V. Teplitz, and I. Tibuleac, astro-ph/0205089.

[18] A.J. Baltz et al., Phys. Lett. B 325, 7 (1994).

[19] D. Beavis et al., Phys. Rev. Lett. 75, 3078 (1995).

[20] A. Rusek et al., Phys. Rev. C 54, R15 (1996).

[21] G. Van Buren et al., J. Phys. G 25, 411 (1999).

[22] Z. Xu et al., J. Phys. G 25, 403 (1999).

[23] T.A. Armstrong et al., Phys. Rev. C 63, 054903 (2001).

[24] J. Barrette et al., Phys. Lett. B 252, 550 (1990).

[25] M. Weber et al., J. Phys. G 27, 487 (2001).

[26] J. Schaffner, C. Greiner, and H. Stocker, Phys. Rev. C 46, 322 (1992). 
[27] J. Schaffner, C.B. Dover, A. Gal, C. Greiner, D.J. Millener, and H. Stöcker, Ann. Phys. (N.Y.) 235, 35 (1994).

[28] J. Schaffner, C.B. Dover, A. Gal, C. Greiner, and H. Stöcker, Phys. Rev. Lett. 71, 1328 (1993).

[29] A. De Rujula, S.L. Glashow, and U. Sarid, Nucl. Phys. B333,
173 (1990).

[30] S. Dimopoulos, D. Eichler, R. Esmailzadeh, and G.D. Starkman, Phys. Rev. D 41, 2388 (1990).

[31] A. Kudo and M. Yamaguchi, Phys. Lett. B 516, 151 (2001).

[32] R. Sayer, Rev. Phys. Appl. 12, 1543 (1977). 